\title{
Reducción de Tiempo de Ciclo en Estación de Trabajo de Industria Automotriz
}

\author{
Laura Viviana Ramírez Gil \\ Karla Gabriela Gómez Bull \\ Mayra Verónica Linares Gil \\ María Marisela Vargas Salgado \\ UACJ-CU \\ Av. Del Desierto 18100 \\ Cd. Juárez s/n, México. \\ Universidad Autónoma de Ciudad Juárez
}

\section{Resumen}

Se trata de la reducción de tiempo de ciclo en la estación de trabajo \#2 de una industria maquiladora del ramo automotriz. Esto generaba un cuello de botella y tiempo muerto en las estaciones subsecuentes por la falta de piezas. Se identificaron los principales desperdicios encontrados y aquellas actividades que no agregaban valor al producto. Se retiraron materiales que se encontraban originalmente dentro de la máquina, se colocaron en un contenedor de forma externa, lo que evitó la interrupción y reinicio de la máquina cada que comenzaba un nuevo ciclo. Se logró reducir el tiempo de ciclo en un 57.69\% con respecto a los tiempos originales. A través del rediseño en la estación mencionada se logró reducir los tiempos de ciclo de la misma.

Palabras clave: Desperdicios, tiempo de ciclo, proceso.

\section{Introducción}

En la industria maquiladora uno de los principales problemas que presentan es el exceso de tiempo en el proceso de producción, el cual ocasiona una mala distribución de la planta, que conlleva a un inadecuado balance en las cargas de trabajo en los empleados, así como el excesivo tiempo de recorrido del material. Una industria que tiene un elevado índice de tiempo en el proceso productivo minimiza su competitividad en el mercado (Del Solar, Sarnillán, Vásquez, \& Fuentes, 2014).

El entorno industrial de la actualidad se caracteriza por la competitividad, la velocidad de los cambios y la inestabilidad de la demanda, esto se debe al incremento que se está presentando por parte de los 
clientes, que requieren productos que se ajusten a sus requerimientos y con la calidad exigida para cumplir con las necesidades especificadas, así mismo el aumento en la rapidez y frecuencia de las entregas (Tejeda, 2011).

En los últimos años, la fabricación de productos como los automóviles, computadoras y electrodomésticos que se fabrican en masa, está teniendo un aumento en la variedad de productos, por lo cual las empresas han adoptado la manufactura esbelta, el objetivo de las industrias es el mejorar la producción, ser rentables, aprovechar al máximo los recursos disponibles, el conjunto de estas herramientas y técnicas representa una oportunidad para el desarrollo de la productividad en la industria actual (Gregorio, Muñoz, Salcedo, \& Sossa, 2011).

En cierta empresa de la localidad dedicada al ramo automotriz se tenía la necesidad de reducir el tiempo de ciclo y los desperdicios generados en la línea de producción, ya que esto impacta directamente en la fecha de entrega del producto, en la satisfacción de cliente, cumplimiento de la demanda, cumplimiento de los requerimientos, en la calidad que se ofrece, ya que el proceso por el que pasa la pieza debe ser el mejor y encontrarse en buenas condiciones, para que sea eficaz y el producto salga con cero defectos.

El presente trabajo contiene información sobre el rediseño realizado en la estación \#2 donde se procesa un condensador para automóvil, con el cual se buscó disminuir el tiempo de proceso del ensamble de los accesorios, ya que, al ser la segunda estación de cuatro, se creaba un cuello de botella por el exceso de tiempo que le tomaba al empleado el ensamblar los accesorios requeridos para pasar la pieza a la siguiente estación, además el operador manifestaba dificultades al tomar y ensamblar los componentes requeridos.

\section{Metodología}

\section{Diseño del Estudio}

Esta investigación es de tipo descriptivo, experimental y longitudinal. Se observaron las variables que intervenían en el tiempo de ciclo para analizar la situación, se trató de modificar los tiempos de ciclo, jugar con los cambios propuestos para reducir el tiempo de ciclo. Se tomaron los datos [tiempos] en un determinado periodo de tiempo, interviniendo con una modificación, para nuevamente monitorear los tiempos $\mathrm{y}$ determinar si verdaderamente se tuvo una mejora (Grajales, 2000).

\section{Materiales}

Entre los materiales utilizados para este trabajo se encuentran: un cronómetro, que se utilizó para tomar el tiempo que tardaba el operador en realizar su operación en la estación; un celular de la marca Lanix ${ }^{\circledR}$ con el cual, se tomó video para estudiar y analizar más a detalle las actividades del trabajador; además se hizo uso del software Variation of Process Time ${ }^{\circledR}$, en el cual se introducía el tiempo que se tomaba con el cronómetro y de esta forma observar si había alguna razón en específico por la cual el proceso de ciclo era más prolongado. 
De igual manera se utilizó un juego de estudios para el análisis de productividad, dichos estudios sirven para encontrar el error del proceso, en este caso el cuello de botella, para así encontrar diferentes mejoras y elegir la que mejor convenga.

\section{Método}

El método para seguir y llevar a cabo el presente proyecto se dividió en tres partes; la primera correspondió a la toma de datos para determinar la situación en la que se encontraba actualmente la estación de trabajo, la segunda fase correspondió a las propuestas de mejora y la última la implementación de las mismas.

A) Toma de datos: Se procedió a tomar tiempos del ciclo a través de un cronómetro, se tomaron las lecturas durante una hora en el primer turno, se iban capturando en el software Variation of Process Time ${ }^{\circledR}$, en el cual se documentó el tiempo de cada ciclo, en algunas ocasiones también se registró la variable por el cual la operación tardaba más del tiempo especificado, al mismo tiempo que se documentaban los tiempos se tomó un video con un celular Lanix ${ }^{\circledR}$ para analizar más a fondo la operación y comenzar a planear posibles mejoras para eliminar el cuello de botella.

B) Propuestas de Mejora: Una vez que se analizaron los tiempos documentados, se observó en el video el método utilizado para la operación, y cuáles fueron las variables que más se hicieron frecuentes a lo largo del proceso, se procedió a realizar propuestas de mejora, para lo cual se observó el área de la estación para conocer el estado en el que se encontraba y de esta forma poder detectar desperdicios. Enseguida se realizó un análisis de la actividad, en donde se registraron los desperdicios encontrados en el área, y a qué clasificación de desperdicio pertenecía, indicando también si era algo que se podía eliminar, reemplazar, entre otras opciones, así mismo cuales eran las posibles soluciones que se podrían implementar.

Luego se elaboró una lista de sugerencias, en donde se anotaron las posibles propuestas de soluciones para cada defecto que fue previamente, en esta hoja se registraba el nombre de la persona que quedaba como responsable de llevar a cabo la mejora y cuánto tiempo estaría a prueba dicha propuesta.

Ya que se tenían las posibles propuestas de mejora, se realizó una lista donde se incluía el problema encontrado, la causa de éste, la acción correctiva que se tomó para solucionarlo, el responsable de llevarla a cabo y además se indica si esta actividad fue funcional o no para la mejora del problema.

c) Implementación de la Mejora: Se procedió a remover el exceso de materia prima de la estación, para lo cual se colocó un cofre en donde fue colocada la misma. Una vez implementada esta mejora, se procedió nuevamente a la toma de tiempos [durante una hora] del proceso, para determinar si la mejora funcionó. 


\section{Resultados}

Con respecto a la toma de tiempos de ciclo en la estación antes de realizar la mejora, se pudo observar que la media de los mismos fue de 46.379 segundos, con una desviación estándar de 10.579, como se puede apreciar en la figura 1. Luego de haber tomado dichos datos se prosiguió a identificar los desperdicios dentro del área, entre los que se encontraron: basura, carros mal acomodados, exceso de materia prima, piezas de proceso en el piso, material fuera de proceso, sobreproducción y material dentro de la máquina.



Figura 1. Tiempos de ciclo antes de la mejora

Se llevó a cabo un plan de acción, en el cual se describen las tareas sobresalientes del taller de productividad para ser implementadas en la estación. A una persona se le asignó la tarea de dar instrucciones al materialista para solo tener en la estación la materia que se necesaria y solo abastecer conforme se vaya requiriendo, mientras que a la persona 2 se le pidió que se diseñaran más racks, a la persona 3 se le asignó la tarea de tomar tiempos, realizar un estudio para balancear los mismos en las 4 estaciones y a la persona
4 se le dio la tarea de diseñar un contenedor para colocar el material de la estación 2 fuera de la máquina.

En el caso del exceso de materia prima, se encontró que la causa era la falta de control en el proceso, la acción propuesta consistió en dar instrucciones al materialista para el abastecimiento de material. En el caso de las piezas en el piso, la causa fue por falta de racks y la acción correctiva fue el diseñar más de éstos. En el desperdicio correspondiente a la sobreproducción en la estación, la causa fue que existía un cuello 
de botella en la estación subsecuente, por lo que la acción que se tomó fue modificarla y balancear las 4 estaciones. Por último, en el caso del material dentro de la máquina, la causa fue que la estación tuvo un mal diseño y la acción que se llevó a cabo fue el diseñar un contenedor para colocar el material fuera de la máquina para que de esta manera no se interrumpiera el ciclo y así evitar un cuello de botella.

Luego de haber tenido dicho estudio se procedió a realizar la mejora y colocar el contenedor de materia prima fuera de la estación de trabajo, para que de esta forma el operador no tuviera que introducir la mano dentro de la máquina y por seguridad, no se interrumpiera el ciclo y tener que volver a iniciarlo cada vez que se comenzará uno nuevo o pasará una nueva pieza, luego de implementar la mejora se procedió a realizar nuevamente otro estudio como el anterior para ver los resultados y se logró una reducción en los tiempos de ciclo. Los nuevos tiempos de ciclo se pueden observar en la figura 2, dichos tiempos ahora tienen una media de 26.897 segundos, es decir el promedio de los tiempos se redujo en un $57.69 \%$ con respecto al tiempo original que se tenía de 46.379 segundos.

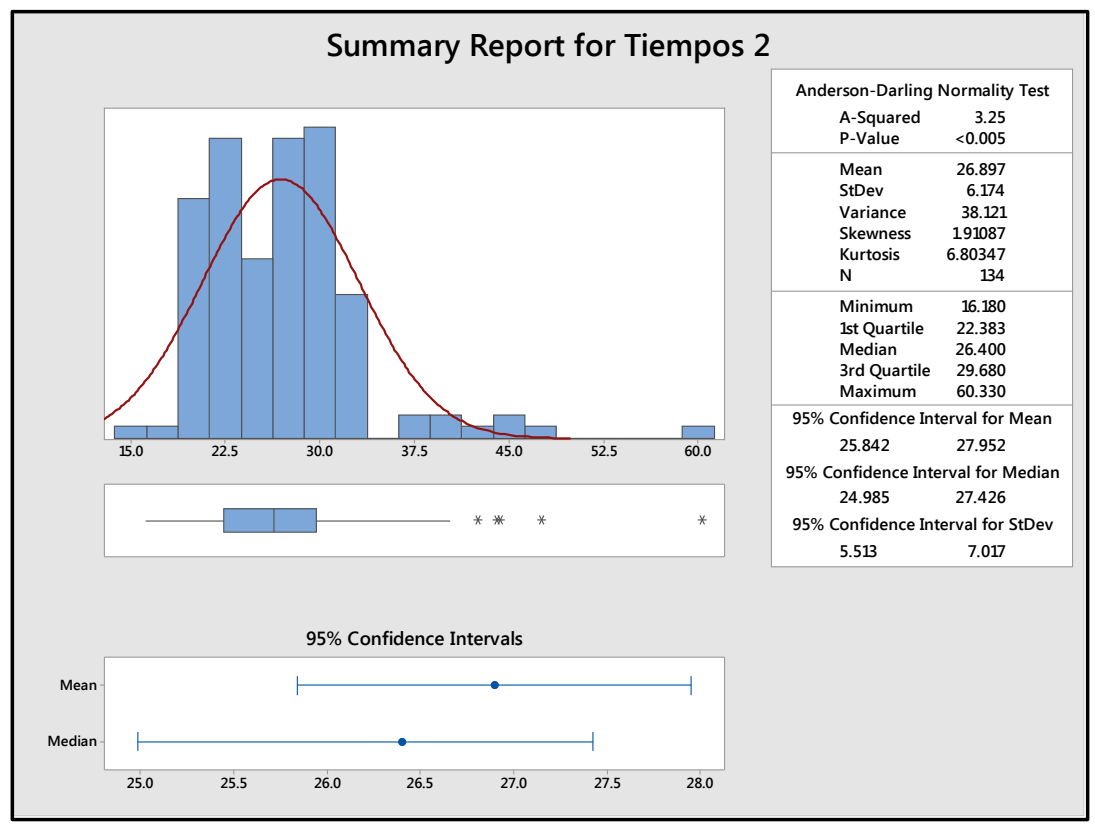

Figura 2. Tiempos de ciclo después de la mejora 


\section{Conclusiones}

Este proyecto tuvo como objetivo general eliminar el cuello de botella generado en una estaciónd e trabajo, el cual se logró a través del rediseño de la misma para que de esa manera la operación fuera más práctica y ágil para el operador, permitiendo el balanceo de la estación y manteniendo el mismo tiempo de ciclo en las siguientes tres estaciones, se logró a través de la toma de tiempos, realizando una serie de estudios, como el Análisis de productividad, en donde se estudió el área en conjunto para de esta forma encontrar la falla, los desperdicios que se tenían, operaciones que se encontraban fuera de proceso, haciendo de esta forma más fácil la eliminación del cuello de botella.

Se pudo observar que es necesario el tener siempre las 5'S como una prioridad, ya que ayudan a mantener el área en buenas condiciones y con esto poder trabajar en una estación adecuada y sin complicaciones por tener acumulamiento de material, basura, cajas vacías, material que no pertenece a esa área, porque los desperdicios y actividades que no agregan valor al proceso son problemas para el proceso y así mismo para la producción. El brindar el entrenamiento adecuado también es vital para que no se presenten incidentes 0 trabajos mal realizados que impliquen retrabajo o la generación de scrap, lo que menos se busca en una empresa es la pérdida y cuando no se da el entrenamiento adecuado, como el leer $\mathrm{y}$ entender las instrucciones de trabajo y ayudas visuales, llevan a la consecuencia de no realizar la operación de forma correcta y esto puede generar la pérdida de tiempo y piezas.

Para seguir mejorando esta área de trabajo, se recomienda brindar la capacitación o entrenamiento adecuado y constante a los operadores, supervisores y técnicos para mantener el área en óptimas condiciones siguiendo y apegándose a las bases de las 5 'S, de igual manera el rediseñar las estaciones de trabajo contemplando antes que nada la seguridad del operador.

\section{Bibliografía}

Colunga C \& A Saldierna. 1994. Costos de la calidad. San Luis Potosí: Universidad Autónoma de San Luis Potosí, Facultad de ingeniería

Fisher M. 1999. Process improvement by pokayoke. Emerald insight, 264-266.

George ML. 2002. Lean six sigma: combining six sigma Quality with lean speed. USA: McGraw- Hill

Gutiérrez H. 2005. Calidad Total y Productividad. México: McGraw-Hill.
PDCA. 2017. PDCA HOME. 13 de Febrero de 2017. http://www.pdcahome.com/poka-yoke/

Rodríguez E. 2005. Metodología de la investigación. Villahermosa: Universidad Autónoma de Tabasco.

Sosa D. 2002. Conceptos y herramientas para la mejora continua. México: Limusa

Soto DM. 2011. Perspectiva de la gestión de la innovación desde los mecanismos a prueba de falla Poka-Yoke. Escenarios. Vol 9, No.1 